\section{Management of failed oral fibreoptic intubation with laryngeal mask airway insertion under topical anaesthesia}

To the Editor:

Awake nasal or oral intubation of the trachea under fibreoptic guidance is accepted as a safe and effective method in cases of suspected difficult intubation. During nasal surgery an oral tube is required and the technically more difficult oral approach must be used.

We recently encountered such a problem in a patient with severe ankylosing spondylitis listed for nasal polypectomy and bilateral antral washouts. After im premedication with papaveretum $15 \mathrm{mg}$ and hyoscine $0.3 \mathrm{mg}$ and $i v$ sedation with droperidol $10 \mathrm{mg}$ and alfentanil 0.5 $\mathrm{mg}$, his tongue and oropharynx were sprayed with lidocaine $10 \%$ (total dose $100 \mathrm{mg}$ ) and $2 \mathrm{ml}$ lidocaine $4 \%$ was injected via cricothyroid puncture. Oral intubation with fibreoptic guidance failed as it proved impossible to enter the anteriorly placed larynx.

Therefore a size \#4 laryngeal mask airway (LMA) was inserted and its cuff inflated which produced a clear airway with no patient discomfort. Anaesthesia was induced with thiopentone $15 \mathrm{mg}$ and maintained with $30 \%$ oxygen in $70 \%$ nitrous oxide and halothane $1-1.5 \%$ with spontaneous ventilation. An oropharyngeal pack was inserted and Moffett's solution instilled into the nasal cavities.

At the end of surgery the pack was removed under direct vision. No blood was seen in the oropharynx. The LMA was left in situ until rejected spontaneously by the patient. At no time was the peripheral oxygen saturation less than $98 \%$.

Although the LMA is known not to protect the larynx from reflux of gastric contents ${ }^{1}$ it has been shown to prevent dye placed in the oropharynx from entering the larynx. $^{2}$

This is the second case reported ${ }^{3}$ describing LMA insertion under topical anaesthesia and sedation. It is a useful technique to employ when presented with abnormal approach to the larynx as the LMA can secure the airway from before the loss of consciousness until recovery of upper airway reflexes. Alternatively, the LMA can be used to aid awake tracheal intubation by guiding a gum elastic bougie ${ }^{3}$ or fibreoptic scope into the larynx.

\section{P.J. Williams BM FRCA}

P.M. Bailey MB BS FRCA
Department of Anaesthesia

The Royal National Throat Nose \& Ear Hospital

Gray's Inn Road

London WC1X 8DA

England

\section{REFERENCES}

1 Brain $A I J$. The Intravent Laryngeal Mask. Instruction Manual. 1990.

2 John RE, Hill S, Hughes TJ. Airway protection by the laryngeal mask. Anaesthesia 1991; 46: 366-7.

3 McCrirrick A, Pracilio JA. Awake intubation: a new technique. Anaesthesia 1991; 46: 661-3.

\section{Patient recruitment for clinical research}

To the Editor:

The following situation illustrates some problems associated with patient recruitment for clinical research that have not been fully addressed.

A randomized study comparing three ocular block techniques for cataract surgery was conducted at our Eye Care Centre (ECC). The protocol of the study dictated that all consecutive patients of the participating surgeon, who were eligible for surgery at the Centre, be included with no exceptions. The patients were made aware of the study when seen by the surgeon in the office, and informed consent was obtained when they arrived at the ECC on the day of surgery.

A 59-yr-old East Indian patient, who did not comprehend English, was accompanied by his son as interpreter. He was in apparent good health. Informed consent was obtained via his son. Before the anaesthetic block was administered, the patient suddenly became unresponsive, with eyes open and glaring upwards, lasting a minute, with no changes in ECG, $\mathrm{BP}$, or $\mathrm{SPO}_{2}$. There was no residual neurological deficit. At the request of the patient, through his son, we proceeded with the block and surgery with no complications. Following several similar episodes of lapses of consciousness on the fourth postoperative day, the patient was admitted to a community hospital. The CT head scan, EEG and 24-hr Holter cardiac monitoring were all normal. The diagnosis was petit mal absence epilepsy.

Several issues arise from this experience. Should surgery have been cancelled? To continue with surgery, especially when the nature of the complication was not clear, might put the study and the patient at further risk. The "no exclusion" clause in the study protocol should not ever have been considered. We proceeded with 
surgery and the study reluctantly upon the insistence of the patient. With the working diagnosis of petit mal absence, the patient was warned of potential dangers. Both anaesthesia and surgery were uneventful, as was his cataract surgery on the other eye seven weeks later.

Ambulatory surgery has become very popular in recent years and many clinical studies in the future will involve day care patients. Anaesthetist-investigators are likely to meet the patients for the first time on the day of surgery. Is informed consent obtained shortly before anaesthesia and surgery adequate? Would initial recruitment by either the surgeon in the office, or an anaesthetic staff (who may not be the investigator) at the preadmission clinic before the actual informed consent have been a more appropriate procedure?

Our ethnic diversity from immigration has resulted in a large portion of the population who do not speak our official languages. The ethical problems of obtaining informed consent, via a third party, from patients who do not comprehend the language need to be addressed.

David H.W. Wong MB BS FRCPC

Department of Anaesthesia

University of British Columbia

Vancouver

\section{Errata}

Re: Guest Reviewers and Translators. Can J Anaesth 1992; 39: 1007-8.

Please note that Dr Martine Pirlet / Sherbrooke was omitted from the list of 'Translators'.

Campbell FA, Yentis SM, Fear DW, Bissonnette B. Analgesic efficacy and safety of a caudal bupivacainefentanyl mixture in children. Can J Anaesth 1992; 39: 661-4.

Please note that the following references were omitted from the original paper.

12 Sevarino $F B, M c F a r l a n e ~ C$, Sinitra $R S$. Epidural fentanyl does not influence intravenous PCA requirements in the post-Caesarian patient. Can J Anaesth 1991; 38: 450-3.

13 Cohen SE, Tan S, Albright GA, Halpern J. Epidural fentanyl/bupivacaine mixtures for obstetric analgesia. Anesthesiology 1987; 67: 403-7.

Re: Asai T. Aspiration and the CMA (1); Shorney N, Brimacombe J. Aspiration and the CMA (2). Can J Anaesth 1992; 39: 746-7.

Please note that "CMA" should read "LMA." 